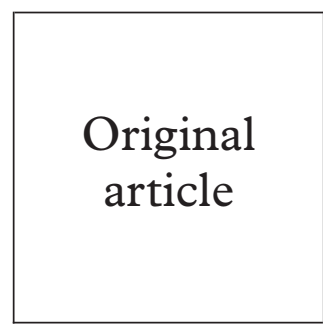

\title{
A colorimetric detection system for Calymmatobacterium granulomatis
}

\author{
Jenny S Carter, David J Kemp
}

Objective: To incorporate the first polymerase chain reaction (PCR) assay for Calymmatobacterium granulomatis into a colorimetric detection system for use in routine diagnostic laboratories. Methods: A capture oligonucleotide specific for the Klebsiella phoE gene was covalently linked to tosyl activated magnetic beads. Biotinylated phoE PCR products obtained from 14 positive specimens from patients with donovanosis and isolates of Klebsiella pneumoniae, $K$ rhinoscleromatis, and Kozaenae were cleaved with HaeIII for the purpose of differentiation, captured by the prepared beads, and subjected to standard EIA detection methodology. Eight samples from unrelated genital conditions underwent the same procedure. It was anticipated from the sequence data that the biotinylated fragment would be cleaved from the capture oligonucleotide target region in the three Klebsiella phoE products (that is, a negative colorimetric result) while the entire fragment of interest would remain intact in the positive $C$ granulomatis $p h o E$ products (that is, a positive colorimetric result).

Results: All 14 positive specimens from patients with donovanosis gave strong colorimetric readings with this detection system. Isolates of $K$ pneumoniae, $K$ rhinoscleromatis, $K$ ozaenae, and the eight specimens from unrelated genital conditions were negative.

Conclusion: The successful development of a colorimetric detection system for $C$ granulomatis incorporating two levels of specificity enables the molecular diagnosis of this condition to be undertaken by routine diagnostic laboratories. This should have an important role in the Australian government's campaign to eradicate donovanosis by 2003 though the test still needs to undergo trials and be validated using a larger number of samples from geographically diverse parts of the world in order to ascertain the generalisability of the methodology.

(Sex Transm Inf 2000;76:134-136)

Keywords: donovanosis; Calymmatobacterium granulomatis; molecular diagnosis; colorimetric detection

Donovanosis is a chronic genital ulcerative disease with endemic foci in socially disadvantaged populations throughout the world. ${ }^{1}$ In recent years there has been a resurgence of interest in this disease owing to the association of genitoulcerative disease with an increased risk of HIV-1 transmission and acquisition. While unproved, there is a highly likely synergistic link between HIV-1 and donovanosis. ${ }^{2}$ The Australian government has a target to eradicate donovanosis by $2003 .^{3}$

The causative organism of donovanosis, Calymmatobacterium granulomatis, has been associated with the genus Klebsiella on the basis of morphological, ultrastructural, and antigenic studies. ${ }^{4}$ Furthermore, we have recently reported phylogenetic evidence for its reclassification as Klebsiella granulomatis comb nov. ${ }^{6}$

Previously we reported the development of the first polymerase chain reaction (PCR) assay for the detection of $C$ granulomatis in clinical specimens. ${ }^{7}$ The specificity of the PCR assay is based on two unique base changes in the $p h o E$ gene of $C$ granulomatis which eliminate HaeIII restriction sites, enabling clear differentiation from closely related species of Klebsiella. A major advantage of this test is that it can be utilised with swab rather than biopsy/tissue smear specimens. It was suggested in this report that it should now be possible to incorporate this PCR/HaeIII digest into a colorimetric detection system for use in routine diagnostic laboratories. We now report a colorimetric detection system for the detection of $C$ granulomatis from clinical specimens. There is no intent to present this work as a validation trial. Rather, our intent is to make this methodology available to as many researchers/ medical practitioners as possible so that appropriate validation trials may be carried out.

DNA extractions and amplification of 188 bp phoE products were carried out as previously reported, ${ }^{7}$ with the exception that a biotin group was attached to the 5' PCR primer. The PCR products were then cleaved by addition of 1 unit of $\mathrm{Hae} \mathrm{III}$ and incubation at $37^{\circ} \mathrm{C}$ for 1 hour. Tosyl activated magnetic beads were obtained (Dynal, Carlton, Victoria, Australia) and washed as recommended by the manufacturer. A capture oligonucleotide with $5^{\prime}$ amine group was designed internal to the HaeIII restriction sites of the $C$ granulomatis phoE product (5' $\mathrm{NH}_{2}$ CCAGCAGGTTCTGATC GTT3') and added to the beads at 150 $\mathrm{pmol} / \mathrm{mg}$ beads in $0.1 \mathrm{M}$ borate buffer $\mathrm{pH} 9.5$. Incubation at $65^{\circ} \mathrm{C}$ for 15 minutes allowed covalent bond formation between the tosyl groups and 5' amine groups. Free tosyl groups were blocked with $0.2 \mathrm{M}$ ethanolamine $\mathrm{pH} 7.0$ containing $0.1 \%$ bovine serum albumin for 4 hours at $37^{\circ} \mathrm{C}$. Before use the beads were diluted 1 in 4 with Probe Suspension 2, pH 4.0 (sodium phosphate solution containing $<0.2 \%$ solubiliser and $<26 \%$ chaotrope, contained within Cobas Amplicor Chlamydia trachomatis Detection Kit, Roche Diagnostic Systems, Inc, 

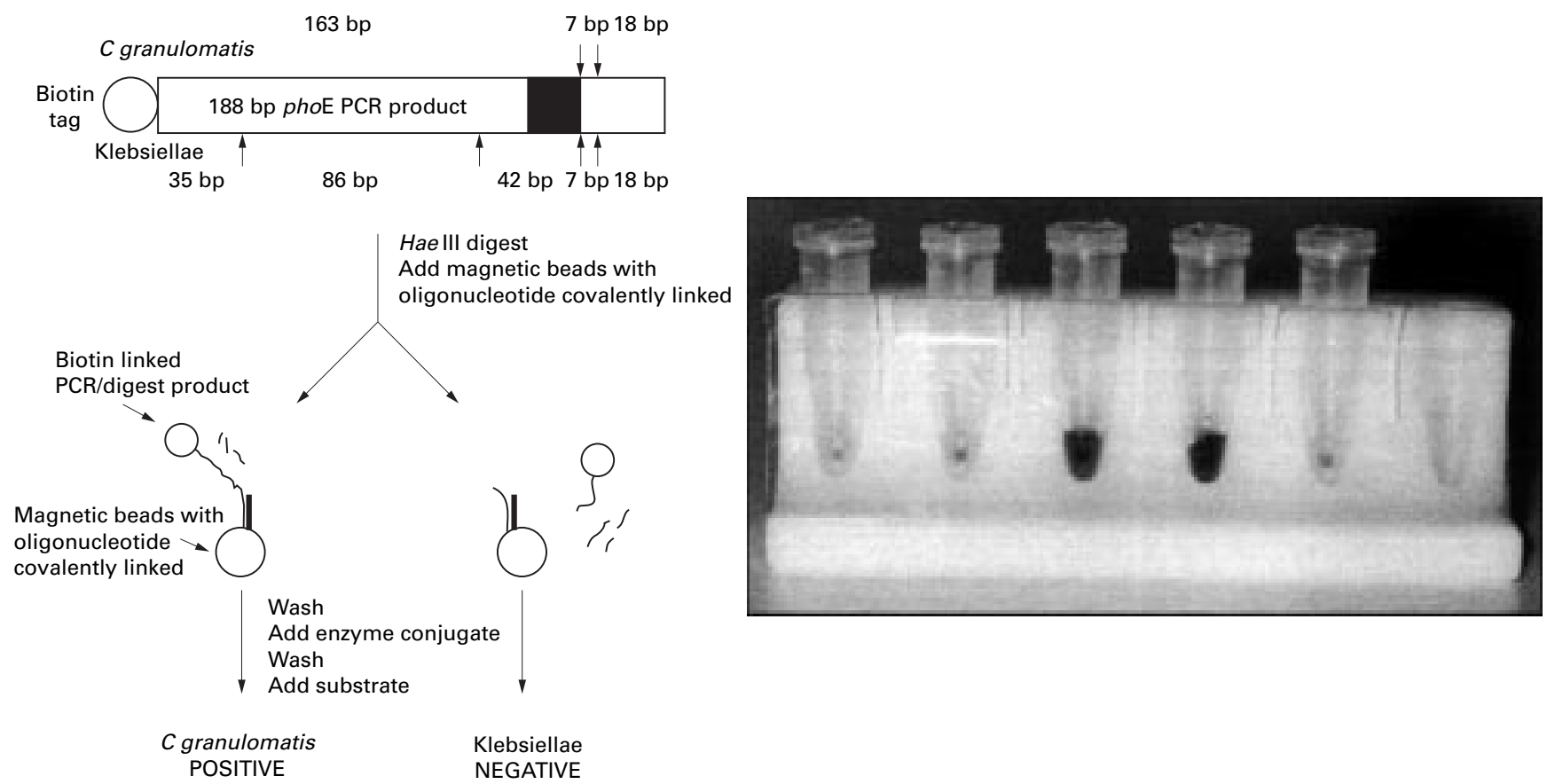

Figure 1 Left: Schematic representation of the colorimetric detection system for $C$ granulomatis. Arrows represent HaeIII restriction sites within the 188 bp phoE product for $C$ granulomatis (above) and other klebsiellae (below). The black box within the 188 bp product represents the target region for the capture oligonucleotide. Right: Results from left to right: K pneumoniae; K rhinoscleromatis; positive swab sample from patient with donovanosis; positive swab sample from patient with donovanosis; K ozaenae.

Branchburg, NJ, USA). PCR/HaeIII digest products $(25 \mu \mathrm{l})$ were denatured with $0.1 \mathrm{M}$ sodium hydroxide $(25 \mu \mathrm{l})$. The denatured solution $(30 \mu \mathrm{l})$ was incubated with $100 \mu \mathrm{l}$ of prepared beads for 30 minutes at $37^{\circ} \mathrm{C}$ to allow for neutralisation and binding with the capture oligonucleotide. All subsequent steps of detection were carried out using the standard EIA methodology (that is, wash, add avidin-HRP conjugate, wash, add substrate). A magnetic particle concentrator (Dynal, Carlton, Victoria, Australia) was utilised at each step to separate the beads from the supernatant and allowed all steps to be carried out in one tube.

A schematic representation of this system is provided (fig 1).

The sample set tested with this detection system was identical to that previously reported, ${ }^{7}$ and included 14 positive samples from patients with donovanosis, eight samples from patients with unrelated genital conditions (herpes genitalis, syphilis, gonorrhoea, human papillomavirus infection, and mixed anaerobic infection - there is no reported chancroid or lymphogranuloma venereum in the Northern Territory of Australia), and isolates of $K$ pneumoniae, $K$ ozaenae, and $K$ rhinoscleromatis. Owing to the cleavage of the fragment containing the biotin group from the capture oligonucleotide target region it was expected that the three Klebsiella digest products would give negative readings while the intact $C$ granulomatis fragment would give positive readings.

Strong colorimetric readings were obtained for all 14 positive samples from patients with donovanosis. All eight samples from patients with unrelated genital conditions were negative as were PCR digests obtained from the three Klebsiella isolates (fig 1).

This methodology has advantages over agarose gel electrophoresis in that it can be utilised by routine diagnostic laboratories and incorporates a second level of specificity via the presence of an internal capture oligonucleotide.

All 14 positive samples from patients with donovanosis included in this report were obtained from the Top End of the Northern Territory of Australia over a 2 year period. However, the test has been/is being successfully utilised by doctors in Central Australia, South Australia, and Western Australia (data not shown as ethical clearance applies only to Top End of the Northern Territory) and at the very least should be particularly valuable if there is a serious attempt to eradicate donovanosis in Australia.

It is interesting to note that the base change at position 532 of the coding nucleotide sequence $(\mathrm{C} \rightarrow \mathrm{T})$ results in the amino acid change $\operatorname{Arg} 157 \rightarrow$ Cys 157. Arg157 is located near the top of one of the surface exposed loops in the Klebsiella PhoE protein and corresponds to $\operatorname{Arg} 158$ of the Escherichia coli PhoE protein. ${ }^{8}$ Korteland et al reported that all $E$ coli $\mathrm{K} 12$ strains tested in their laboratory with resistance to the PhoE specific bacteriophage TC45 showed a mutation at $\operatorname{Arg} 158 .^{9}$ While unproved, it is plausible that the $C$ granulomatis isolates present in Australia have also acquired this selective advantage at some point in their evolution.

This gives some strength to the future stability of this mutation in the Australian context though the question still remains as to whether the organism is clonal in an international context. The specificity of the PCR assay still needs to undergo trials and be validated using a larger number of samples from geographically diverse locations within and outside Australia in order to test the stability and applica- 
bility of the two unique base changes on which it relies.

Jenny S Carter is in receipt of a scholarship from the National Health and Medical Research Council of Australia.

Contributors: JSC contributed to the methodological design and strategic direction of the research presented, carried out the laboratory work, and wrote the first and subsequent drafts of the manuscript; DJK contributed to the methodological design and strategic direction of the research presented, was directly responsible for the supervision of JSC and provided critical input to the manuscript.

1 Hart G. Donovanosis. Clin Infect Dis 1997;25:24-30.

2 Fleming DT, Wasserheit JN. From epidemiological synergy to public health policy and practice: the contribution of other sexually transmitted diseases to sexual transmission of HIV infection. Sex Transm Inf 1999;75:3-17.

3 Commonwealth of Australia. The National Indigenous Australians' Sexual Health Strategy 1996-97 to 1998-99.
Canberra, ACT: Australian Government Publishing Service, 1997.

4 Kuberski T, Papadimitriou J, Phillips P. Ultrastructure of Calymmatobacterium granulomatis in lesions of granuloma inguinale. F Infect Dis 1980;142:744-9.

5 Packer H, Goldberg J. Studies of the antigenic relationship of D granulomatis to members of the tribe Escherichiae. Am f Syph Gon Vener Dis 1950;34:342-50.

6 Carter JS, Bowden FJ, Bastian I, et al. Phylogenetic evidence for reclassification of Calymmatobacterium granulomatis as Klebsiella granulomatis comb nov. Int 7 Syst Bacteriol 1999;49:1695-700.

7 Carter JS, Bowden FJ, Sriprakash KS, et al. Diagnostic polymerase chain reaction for donovanosis. Clin Infect Dis polymerase chain

8 Van der Ley P, Bekkers A, Van Meersbergen J, et al. A comvar der Ley P, Bekkers A, Van Meersbergen J, et al. A com-
parative study on the phoE gene of three enterobacterial species. Implications for structure-function relationships in a pore-forming protein of the outer membrane. Eur 7 Biochem 1987;164:467-75

9 Korteland J, Overbeeke N, de Graaff P, et al. Role of the Arg158 residue of the outer membrane PhoE protein of Escherichia coli K 12 in bacteriophage TC45 recognition and in channel characteristics. Eur f Biochem 1985;152 $691-7$.

\section{Joint Meeting of MSSVD and the ASTDA}

3-7 May 2000, Baltimore Marriott Inner Harbor Hotel, Baltimore, Maryland, USA

To mark the unique nature of the millennial year, for the first time in its 78 year history the Medical Society for the Study of Venereal Diseases will hold its Spring Meeting jointly with the American Sexually Transmitted Disease Association (President, Professor Julius Schacter). Our local host will be Professor Jonathan Zenilman of Johns Hopkins Medical School. The scientific programme will consist of plenary lectures and round table discussions, delivered by world authorities. There will also be oral and poster presentations of original work.

Further mailings will follow to MSSVD and ASTDA members. People who do not belong to either of these organisations and who would like to receive further information should contact: Dr Keith Radcliffe, Honorary Assistant Secretary, MSSVD (fax: +44 (0) 121-237 5729; email: k.w.radcliffe@bham.ac.uk). 